\title{
Increased sensitivity to SMAC mimetic LCL161 identified by longitudinal ex vivo pharmacogenomics of recurrent, KRAS mutated rectal cancer liver metastases
}

\author{
Kushtrim Kryeziu1, Seyed H. Moosavi 1,2, Christian H. Bergsland ${ }^{1,2}$, Marianne G. Guren ${ }^{3}$, Peter W. Eide', \\ Max Z. Totland ${ }^{1}$, Kristoffer Lassen ${ }^{4,5}$, Andreas Abildgaard ${ }^{6}$, Arild Nesbakken ${ }^{2,7}$, Anita Sveen ${ }^{1,2}$ and \\ Ragnhild A. Lothe ${ }^{1,2^{*}}$ (D)
}

\begin{abstract}
Tumor heterogeneity is a primary cause of treatment failure. However, changes in drug sensitivity over time are not well mapped in cancer. Patient-derived organoids (PDOs) may predict clinical drug responses ex vivo and offer an opportunity to evaluate novel treatment strategies in a personalized fashion. Here we have evaluated spatio-temporal functional and molecular dynamics of five PDO models established after hepatic re-resections and neoadjuvant combination chemotherapies in a patient with microsatellite stable and KRAS mutated metastatic rectal cancer. Histopathological differentiation phenotypes of the PDOs corresponded with the liver metastases, and ex vivo drug sensitivities generally reflected clinical responses and selection pressure, assessed in comparison to a reference data set of PDOs from metastatic colorectal cancers. PDOs from the initial versus the two recurrent metastatic settings showed heterogeneous cell morphologies, protein marker expression, and drug sensitivities. Exploratory analyses of a drug screen library of 33 investigational anticancer agents showed the strongest ex vivo sensitivity to the SMAC mimetic LCL161 in PDOs of recurrent disease compared to those of the initial metastasis. Functional analyses confirmed target inhibition and apoptosis induction in the LCL161 sensitive PDOs from the recurrent metastases. Gene expression analyses indicated an association between LCL161 sensitivity and tumor necrosis factor alpha signaling and RIPK1 gene expression. In conclusion, LCL161 was identified as a possible experimental therapy of a metastatic rectal cancer that relapsed after hepatic resection and standard systemic treatment.
\end{abstract}

Keywords: Rectal cancer, Liver metastasis, Patient-derived organoid, LCL161

\section{Introduction}

"Living biobanks" of patient-derived tumor organoids (PDOs) can model histopathological and pharmacogenomic heterogeneity in cancer [1-5]. Co-clinical studies have shown that PDOs can predict clinical responses to

*Correspondence: ragnhild.a.lothe@rr-research.no

1 Department of Molecular Oncology, Institute for Cancer Research, Oslo University Hospital, Nydalen, P. O. Box 4953, 0424 Oslo, Norway

Full list of author information is available at the end of the article both targeted agents and chemotherapies in an observational setting $[6,7]$. However, tumor heterogeneity is a major driving force of treatment failure [8]. PDOs established from distinct liver lesions of patients with metastatic colorectal cancer (CRC) have shown that inter-metastatic heterogeneity of drug sensitivities is limited [9], but systemic anticancer treatment inflicts selective pressures that can drive the evolution of drugresistant subclones [10-13]. Longitudinal monitoring may therefore guide treatment adaptation, as illustrated original author(s) and the source, provide a link to the Creative Commons licence, and indicate if changes were made. The images or other third party material in this article are included in the article's Creative Commons licence, unless indicated otherwise in a credit line to the material. If material is not included in the article's Creative Commons licence and your intended use is not permitted by statutory regulation or exceeds the permitted use, you will need to obtain permission directly from the copyright holder. To view a copy of this licence, visit http://creativecommons.org/licenses/by/4.0/. The Creative Commons Public Domain Dedication waiver (http://creativeco mmons.org/publicdomain/zero/1.0/) applies to the data made available in this article, unless otherwise stated in a credit line to the data. 
by rechallenge with anti-EGFR therapy against metastatic CRC guided by monitoring of KRAS mutation levels in the blood $[14,15]$. However, most studies of cancer cell drug vulnerabilities and functional modeling are crosssectional and provide 'snapshots' of a single point in time $[1,2,7,16]$.

Patients diagnosed with KRAS mutated CRC have limited systemic treatment options when resistance to standard combination chemotherapies with or without antiangiogenic agents occurs $[17,18]$. The mechanisms of chemoresistance in KRAS-driven cancers are not well understood. However, acquired chemoresistance might be associated with evasion of apoptosis via dysregulated expression of inhibitor of apoptosis proteins (IAPs) [19, 20]. IAPs can directly inhibit caspases or divert death-inducing signals of the tumor necrosis factor (TNF) pathway into prosurvival signals via activation of the proliferative transcriptional programs of Receptorinteracting protein kinase 1 (RIPK1) and Nuclear factor kappa-B (NF-kB) [19, 21-23]. Endogenous regulation of IAPs is mediated by second mitochondria-derived activator of caspases (SMACs), which are released from the mitochondria into the cytosol in response to apoptotic stimuli, and thus free the caspases to execute pro-apoptotic functions. Small therapeutic compounds mimicking SMACs can promote apoptosis in cancers with deregulated IAP expression [19]. However, the clinical success of SMAC mimetics as anticancer agents has been limited by the lack of predictive biomarkers [24].

Here, we report a longitudinal, observational co-clinical study of standard combination chemotherapies in a patient with recurrent, KRAS mutated liver metastases from rectal cancer. A SMAC mimetic was identified as a novel therapeutic opportunity in PDOs of the recurrent tumors.

\section{Materials and methods Patient and samples}

The patient diagnosed with metastatic rectal cancer was surgically treated for liver metastases at Oslo University Hospital on three occasions, between December 2017 and February 2020. Parallel samples from resected specimens were fresh frozen $\left(-80{ }^{\circ} \mathrm{C}\right)$ for molecular profiling, or transported ice-cold in basal media (Advanced DMEM/F-12 supplemented with $10 \mathrm{mM}$ HEPES, $2 \mathrm{mM}$ GlutaMAX all from Thermo Fischer Scientific and $100 \mu \mathrm{g} / \mathrm{ml}$ Primocin from Invivogen) for organoid cell culturing within $24 \mathrm{~h}$. A sample set of similarly processed liver metastases $(n=46)$ from 23 patients diagnosed with metastatic CRC described in ref [9] have been used as the reference dataset in this study.

\section{Culturing patient-derived tumor organoids}

Samples from resected tumor specimens $(2.5-6 \times 7 \mathrm{~mm}$ in size) were minced into $0.1-0.5 \mathrm{~mm}$ fragments, washed with ice-cold basal culture media, strained with a $70 \mu \mathrm{m}$ pore mesh, and collected by centrifugation at $400 \mathrm{~g} 4{ }^{\circ} \mathrm{C}$ for $5 \mathrm{~min}$. The pellet was suspended in Growth Factor Reduced Matrigel (Corning), dispensed onto pre-warmed 6-well tissue culture plates as $25 \mu \mathrm{l}$ drops, overlaid with $3 \mathrm{ml}$ organoid growth media supplemented with $10 \mu \mathrm{M} \mathrm{Y}-27632$ and then incubated at $37{ }^{\circ} \mathrm{C}$ in a humidified $5 \% \mathrm{CO}_{2}$ atmosphere. Organoid growth media consisted of basal culture media supplemented with 1xB27 supplement (Gibco), $10 \mathrm{nM}$ [Leu15]-Gastrin I (Sigma) and $1 \mathrm{mM} \mathrm{N}$-acetyll-cysteine (Sigma) and the following niche factors: $50 \mathrm{ng} / \mathrm{mL}$ for EGF (Gibco), $100 \mathrm{ng} / \mathrm{mL}$ for Noggin (Preprotech), $500 \mathrm{nM}$ for TGF- $\beta$ receptor type I inhibitor A83-01 (Tocris) and $10 \mu \mathrm{M}$ for $\mathrm{p} 38$ MAP kinase inhibitor SB202190 (Sigma). Organoid growth media without Y-27632 was refreshed every two to four days. Organoids were passaged by digestion with TrypLE Express (Gibco) for 5 min at $37^{\circ} \mathrm{C}$ supplemented with $10 \mu \mathrm{M}$ Y-27632. Contamination-free organoid cultures were ensured using MycoAlert Mycoplasma Detection Assay (Lonza) within a week after the functional assays or before cryopreservation. The authenticity of the cultures was verified by comparison to the respective tumor tissues using AmpFLSTR Identifiler PCR Amplification Kit (Thermo Fisher Scientific).

\section{Drug sensitivity screening}

A medium throughput drug screen of a custom library of 33 clinically relevant small molecule inhibitors in eight different concentrations each, and three drug combinations in seven concentrations was performed as previously described [9]. The drug library was selected for clinical relevance in CRC, and included all small molecules approved to treat CRC, drugs with emerging clinical evidence of activity against CRC, and selected drugs that are either approved or in clinical testing for other cancer types. Selection among agents with the same mechanism of action was based on robust drug sensitivity scores tested in CRC cell lines [25] and the furthest development in clinical studies. The setup of one treatment reaction included 40 fold drug concentration (preprinted with liquid acoustic dispensing technology Echo 550, Labcyte Inc. at the 
High Throughput Biomedicine Unit at the Institute for Molecular Medicine Finland) overlaid with $10 \mu \mathrm{l}$ of $50 \%$ Matrigel, followed by a suspension of 450-600 prestrained organoids (70 $\mu \mathrm{m}$ mesh size) with $30 \mu \mathrm{l}$ of $3 \%$ Matrigel. Two parallel replicas per sample were incubated with the drugs and positive $(100 \mu \mathrm{M}$ benzethonium chloride, $\mathrm{n}=9$ wells) and negative (0.1\% DMSO, $\mathrm{n}=13$ wells) controls for $96 \mathrm{~h}$ at $37{ }^{\circ} \mathrm{C}$ in a humidified $5 \% \mathrm{CO}_{2}$ atmosphere, and analyzed by the CellTiterGlo 3D Cell Viability Assay (Promega) according to the manufacturer's instructions, prior to luminescence measurement with a Victor 3 microplate reader (Perkin Elmer). Luminescence readouts were rescaled to relative viability based on the median of the negative and positive control wells per plate (separately for the two technical replicates). Data from technical replicates were combined to estimate dose-response curves [9]. Drug sensitivity scores were calculated as described in Yadav et al. (2014) [26].

\section{Growth rate adjustment of drug activities}

For growth rate adjusted drug sensitivity scores $\left({ }_{G R} D S S\right)$, area under the curve was calculated using growth rate in place of relative viability based on a previously developed method for estimation of growth fold-changes [27]. To estimate growth rate, the following steps were performed. Micrographs were captured at baseline seeding of each drug screen, as well as after four days of incubation with the negative and positive controls using an EVOS FL microscope (Thermo Fisher Scientific). The volume of more than 150 structures of each sample at baseline, and of 150 structures after $96 \mathrm{~h}$ incubation with the negative control, were measured manually using Image (Fiji) based on the longest (l) and shortest (s) diameters of the structures using the following formula:

$$
V=\frac{\pi l s^{2}}{6}
$$

Volume fold-changes $(\Delta v)$ were calculated as the mean volume of structures after incubation with negative control divided by the volume of the baseline structures, and used to calculate organoid doubling time $(T d)$ as follows:

$$
\Delta \mathrm{v}=2^{\mathrm{Te} / \mathrm{Td}} \rightarrow \mathrm{Td}=\frac{\operatorname{Telog}(2)}{\log (\Delta \mathrm{v})}=\frac{\mathrm{Te}}{\log _{2}(\Delta \mathrm{v})}
$$

where $T e$ is the experimental duration (typically $96 \mathrm{~h}$ ).

Drug screen quality was evaluated based on the strictly standardized mean difference (SSMD) metric of the raw luminescence readouts from DMSO treated (neg) and benzethonium chloride treated (pos) conditions as follows:

$$
S S M D=\frac{\operatorname{mean}(\text { pos })-\operatorname{mean}(\text { neg })}{\sqrt{\operatorname{var}(\text { pos })+\operatorname{var}(\text { neg })}}
$$

Samples with $\mathrm{SSMD}<3$ were discarded and repeated. For the included screens, the median SSMD was 9.3 [range: $3.5-34$ ].

\section{DNA/RNA extractions and mutation analyses}

DNA and RNA were extracted from fresh-frozen tissue samples and PDOs using the Allprep DNA/RNA/miRNA Universal kit (Qiagen GmBH, Hilden, Germany), following the manufacturer's instructions. Microsatellite instability (MSI) status and KRAS, NRAS, BRAF hotspot mutation status were determined as previously described $[9,28]$.

\section{Gene expression analyses}

Gene expression profiles were generated for all resected liver metastasis tissue samples and their corresponding PDOs using the GeneChip Human Transcriptome Array 2.0 (HTA 2.0) with $100 \mathrm{ng}$ of total RNA as input and following the manufacturer's protocol (Thermo Fisher Scientific). Raw intensity data were pre-processed, normalized and $\log 2$ transformed for tissue samples and PDOs separately by robust multi-array average (RMA) method implemented in justRMA function in the R package affy [29] using the custom Entrez CDF file (v24) from Brainarray [30]. Entrez IDs were converted to HGNC gene symbols using the org.Hs.eg.db package (v 3.7.0) from Bioconductor. Single-sample gene set enrichment analyses of gene signatures of response and resistance to 5-fluorouracil (5-FU) [31], as well as the "hallmark" gene sets $(n=50$; retrieved from the Molecular Signatures Database, v7.0 [32]) were performed by the gsva function (gene set variation analysis) in the R package GSVA [33]. Sensitivity to LCL161 was also analyzed in relation to $\log 2$ expression signals of previously suggested target genes encoding mediators of the TNF alpha pathway and apoptosis regulators [34].

\section{Immunostaining}

All PDO lines and one liver metastasis tissue were formalin-fixed, paraffin-embedded and assembled in a microarray with $4 \mathrm{~mm}$ cores, sliced at $3 \mu \mathrm{m}$ sections, and stained for hematoxylin and eosin. A section was also stained and analyzed for caudal type homeobox 2 (CDX2), cytokeratin 20 (CK20), E-cadherin (ECAD) and cytokeratin 7 (CK7) using multiplexed fluorescence staining based on 
Opal kits and reagents (product numbers NEL810001KT and FP1495001KT, Akoya Biosciences) and multispectral imaging (Vectra3 imaging system, Akoya Biosciences). The following antibodies and fluorophores were used to detect each target: anti-CDX2 (1:400, clone EPR2764Y, Cell Marque) detected by Opal 570, anti-CK20 (1:1000, clone Ks20.8, Agilent Dako) detected by Opal 520, antiECAD (1:10.000, clone 36, BD Biosciences) detected by Opal 690, anti-CK7 (1:400, clone OV-TL 12/30, Agilent Dako) detected by Opal 620. Cell nuclei were stained with DAPI. Multispectral images were unmixed in Inform Software (Akoya Biosciences) and all images displayed are scaled equally.

\section{Western Blot analyses}

$7.5 \times 10^{3}$ PDOs were incubated for $48 \mathrm{~h}$ with $200 \mu \mathrm{M}$ LCL161 or $0.01 \%$ DMSO. After drug incubation, the cells were washed with $1 \times$ PBS, scraped, collected, lysed in $100 \mu \mathrm{l}$ SDS electrophoresis sample buffer $(10 \mathrm{mM}$ Tris (pH 6.8), 15\% w/v glycerol, $3 \% \mathrm{w} / \mathrm{v}$ SDS, $0.01 \% \mathrm{w} / \mathrm{v}$ bromphenol blue, and $5 \% \mathrm{v} / \mathrm{v} 2$-mercaptoethanol), sonicated and heated at $95{ }^{\circ} \mathrm{C}$ for $5 \mathrm{~min}$. Protein concentration was determined using the RC-DC Protein Assay kit (BioRad) according to the manufacturer's recommendation. Protein lysates were separated on $12 \%$ SDS-PAGE gels by electrophoresis then blotted onto PVDF membranes using a Trans-Blot ${ }^{\circledR}$ Turbo $^{\text {TM }}$ Transfer System (Bio-Rad). The primary antibodies used were: c-IAP1 (D5G9) Rabbit mAb \#7065, c-IAP2 (58C7) Rabbit mAb \#3130, XIAP (3B6) Rabbit mAb \#2045, Survivin (71G4B7) Rabbit mAb \#2808, Cleaved Caspase-8 (Asp384) (6B6) Mouse mAb \#9747, Caspase-7 (C7) Mouse mAb \#9494, Caspase-3 Antibody \#9662, PARP Antibody Rabbit \#9542, Cleaved PARP (Asp214) (D64E10) XP(R) Rabbit mAb \#5625 all from Cell Signaling at 1:1000 dilution as well as anti- $\beta$ actin \#A2228 from Sigma-Aldrich at 1:5000 dilution. HRP conjugated secondary antibodies (Bio-Rad) were used at 1:5000 dilution and chemiluminescence detected using SuperSignal West Dura Extended Duration
Substrate kit (Thermo Fisher Scientific) on a Bio-Rad image station. Protein band intensities were quantified with Image Lab V5.2 Software from Bio-Rad.

\section{Results}

\section{Case presentation and PDO establishment}

A 64 year old man was diagnosed with KRAS mutated (codon G13D), microsatellite stable rectal cancer disseminated to the liver (T3N0M1; Fig. 1). The patient was scheduled for neoadjuvant combination chemotherapy with 5-FU, leucovorin and oxaliplatin (FLOX) followed by liver resection, and the metastases showed partial response (according to response evaluation criteria in solid tumors 1.1) [35], with a mean shrinkage of the measured tumor deposits of $75 \%(n=5$, range: 45\%-100\%) (Fig. 1 upper left). One lesion was resected (T1: $10 \mathrm{~mm}$ diameter reduction, corresponding to $55 \%$ tumor shrinkage; clear resection margin) and submitted for organoid culturing and genomics [9]. All other lesions were treated with microwave ablation. Histopathological hematoxylin and eosin stains of the PDOs showed three-dimensional cell structures with well- and poorly-defined epithelial layers and smallor absent lumens, indicating a moderately differentiated phenotype (Fig. 1 upper right). ECAD, CDX2, and CK20 were expressed, whereas CK7 was absent, supporting epithelial CRC tissue origin.

The patient remained disease-free until ten metastases were detected at new locations in the liver by magnetic resonance imaging (MRI) at follow-up 11.7 months after surgery. The patient was scheduled for chemotherapy followed by hepatic re-resection, and the recurrent lesions showed partial response to neoadjuvant FLOX (Fig. 1 middle left). All lesions were resected with clear margins, and the two lesions with poorest response to chemotherapy remained amenable to sampling, organoid culturing and genomics (T2: $3 \mathrm{~mm}$ diameter reduction, corresponding to 25\% tumor shrinkage; T3: $2 \mathrm{~mm}$ diameter increase, $11 \%$ tumor growth). Both PDOs showed a

\footnotetext{
(See figure on next page.)

Fig. 1 Clinical history of the patient. One increment in the timeline ruler corresponds to five weeks, and numbers indicate months starting from the MRI before neoadjuvant systemic treatment to surgical interventions. Left column shows MRls (transverse plane) before and after neoadjuvant combination chemotherapy for all three surgeries, and bar graphs indicate radiologic response measurements of individual lesions (T1-T5 are sampled lesions, Tx are non-sampled lesions) as the percent difference in diameter $(\% \Delta \emptyset)$ and as the absolute size difference in $\mathrm{mm}(\Delta \mathrm{mm})$. Columns to the right indicate (from left) chemotherapy (number of cycles and duration), resection of liver metastases, tissue sampling from the indicated liver segment (Roman numeral), immunohistochemistry of diagnostic markers in PDOs and one tissue sample. Scale bar $=50 \mu \mathrm{m}$. FLOX 5-fluorouracil, leucovorin, and oxaliplatin, FLIRI 5-fluorouracil, leucovorin, and irinotecan, PDO patient-derived organoid, LM liver metastasis, NED No evidence of disease, H\&E hematoxylin and eosin
} 


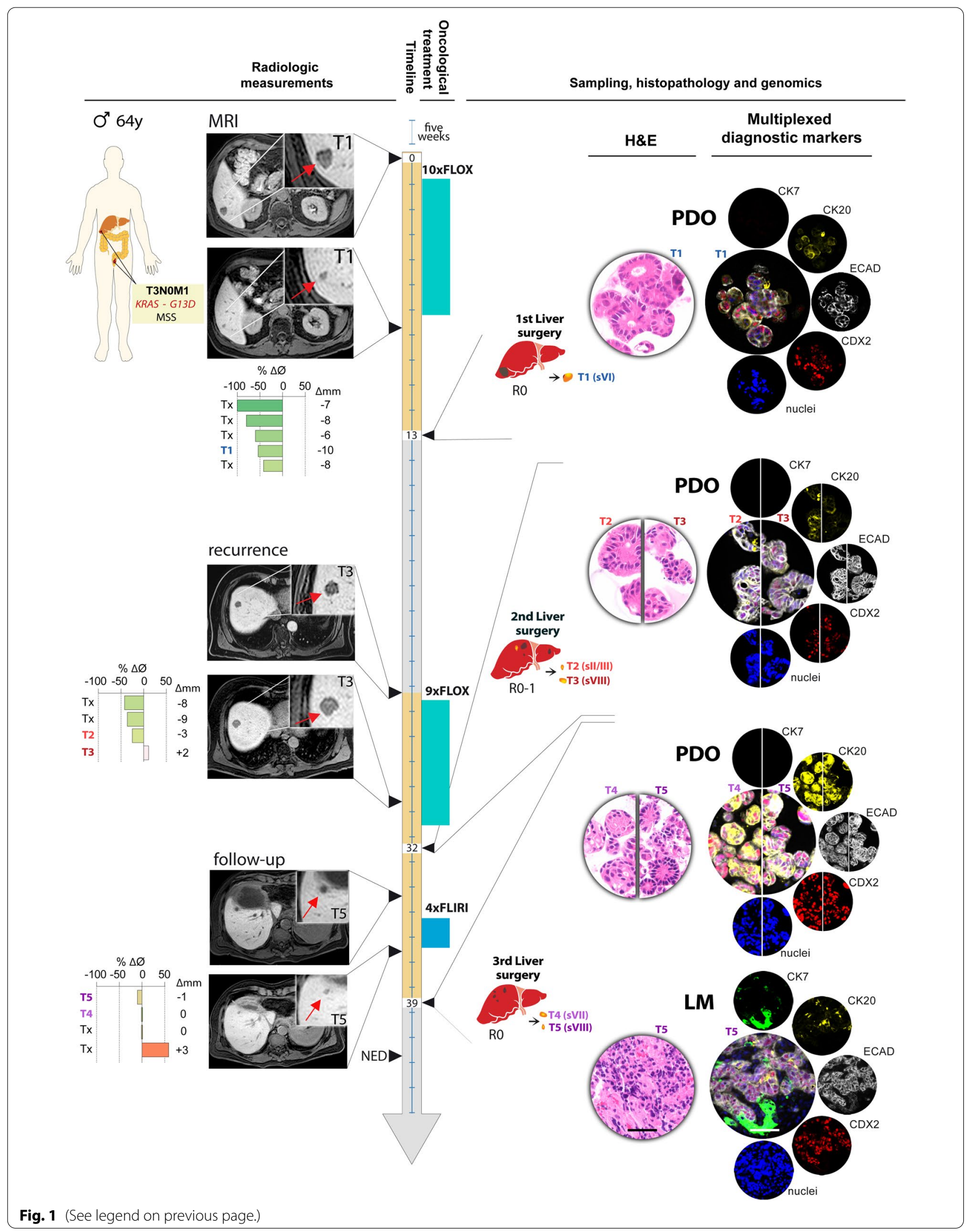


more undifferentiated phenotype compared to the PDO from the first resection, with small or absent lumens, and disorganized epithelial cell layers with ECAD, CDX2 and CK20 expression (Fig. 1 middle right).

Ten weeks after re-resection, one previously undetected metastatic lesion and three new lesions were seen on follow-up MRI. Four cycles of neoadjuvant treatment with 5-FU, leucovorin and irinotecan (FLIRI) resulted in heterogeneous responses among the lesions (Fig. 1 bottom left). Two of the new lesions were resected and submitted for organoid culturing and genomics (T4: no diameter change of the $6 \mathrm{~mm}$ large tumor; T5: $1 \mathrm{~mm}$ diameter reduction corresponding to $9 \%$ tumor shrinkage), and the two other lesions were treated with radiofrequency ablation. Similar to PDOs from the second liver surgery (T2 and T3), PDOs from $\mathrm{T} 4$ and $\mathrm{T} 5$ exhibited an undifferentiated morphology, but with a slightly stronger CK20 and CDX2 expression (Fig. 1 bottom right). For reference, the corresponding tissue sample from the $\mathrm{T} 5$ lesion was stained for the same diagnostic markers, showing that the PDO retained the undifferentiated phenotype and expression patterns of the tumor.

\section{Spatio-temporal co-clinical evaluation of standard chemotherapies}

All PDOs were screened with a customized mediumthroughput drug library incorporating 33 single agents and three 5-FU-based drug combinations with Leucovorin (FLV), Oxaliplatin (FLOX), and SN-38 (FLIRI) (Fig. 2A, B). Co-clinical evaluation of FLOX showed that the PDO from the first resection reflected the strong clinical response in the corresponding $\mathrm{T} 1$ lesion (Fig. 1 upper left and Fig. 2B). The ${ }_{\text {GR }} \mathrm{DSS}$ in the T1 PDO indicated a particularly strong sensitivity to 5-FU, also in comparison to our reference dataset of 46 PDOs from 23 patients with resected CRC liver metastases [9] (Fig. 2A), suggesting that the clinical response was primarily driven by this agent. Sensitivity to 5-FU was much lower in the two PDOs from the second resection, and sensitivity to oxaliplatin was largely unchanged, consistent with the weaker clinical responses to FLOX in the corresponding lesions (T2 and T3). Notably, heterogeneous sensitivity to combination therapies with FLV and FLOX between T2 and T3 PDOs did not correspond to the relative radiological responses of the corresponding lesions (Fig. 2B), potentially related to the poor clinical response of both lesions. T4 and T5 PDOs from the third resection showed higher sensitivity to oxaliplatin and lower sensitivity to FLIRI than PDOs from previous resections, possibly associated with a different selection pressure after the change in treatment from FLOX to FLIRI in the third neoadjuvant setting. 5-FU showed heterogeneous activity between T4 and T5 PDOs, not consistent with the poor clinical responses to FLIRI in corresponding lesions, or the small difference in ex vivo sensitivity to FLIRI between the PDOs.

Across all PDOs and the reference PDO dataset, gene expression signatures of response and resistance to 5-FU [31], analyzed as GSVA scores, were significantly correlated to the measured 5-FU sensitivity (Fig. 2C).

\section{Increased sensitivity to SMAC mimetic LCL161 in recurrent lesions}

Low sensitivity to EGFR inhibitors was confirmed in these KRAS mutated PDOs, both relative to other anticancer agents and relative to $R A S$ wild-type PDOs in the reference dataset (Fig. 2A and D). The highest mean sensitivity score in the five PDOs across the complete tested panel of single agents was found for the SMAC mimetic LCL161 (Fig. 2A). However, most of the drugs showed intra-patient heterogeneity and differential activities among the five PDOs. Largest difference in sensitivity was found for LCL161, showing higher activity in PDOs from the second and third resections, with 5.8 and 4.4-fold higher ${ }_{\text {GR }}$ DSS compared to T1 PDOs, respectively, and far lower $\mathrm{IC}_{50}$ than the maximum plasma concentration of LCL161 [36] (Fig. 3A). The

\footnotetext{
(See figure on next page.)

Fig. 2 Drug sensitivity analyses of a medium throughput drug library. (A, upper panel) ${ }_{G R} D S S$ of 33 drugs in T1-T5 (colored dots) as compared to the reference PDO lines (grey dots, $n=46)$. ( $\left(\mathbf{A}\right.$, lower panel) Mean ${ }_{G R} D S S$ of 33 drugs in T1-T5 centered to the mean of the entire dataset $(n=51)$. The grey line within the bars indicates standard deviation of the respective drug's ${ }_{G R} D S S$ for T1-T5 lesions. B Drug activities of 5-FU, oxaliplatin, SN-38 (active metabolite of irinotecan) as single agents and combination therapies in T1-T5 PDOs. C Scatter plots of 5-FU drug sensitivity scores (growth rate adjusted) and GSVA scores of signatures of 5-FU sensitivity and resistance in PDOs from this patient (colored as indicated) and a reference PDO dataset (grey). D EGFR inhibitor activities and their association with RAS/RAF mutation status analyzed in 23 mutated and 28 wild type PDOs. ${ }_{G R} D S S$ - Growth adjusted drug sensitivity scores, 5-FU 5-fluorouracil, FLV 5-FU + $10 \mu \mathrm{M}$ leucovorin, FLOX 5-FU and oxaliplatin at 1:1 ratios + $10 \mu \mathrm{M}$ leucovorin, FLIRI 5-FU and SN-38 at 100:1 ratios + $10 \mu \mathrm{M}$ leucovorin, GSVA—gene set variation analysis
} 


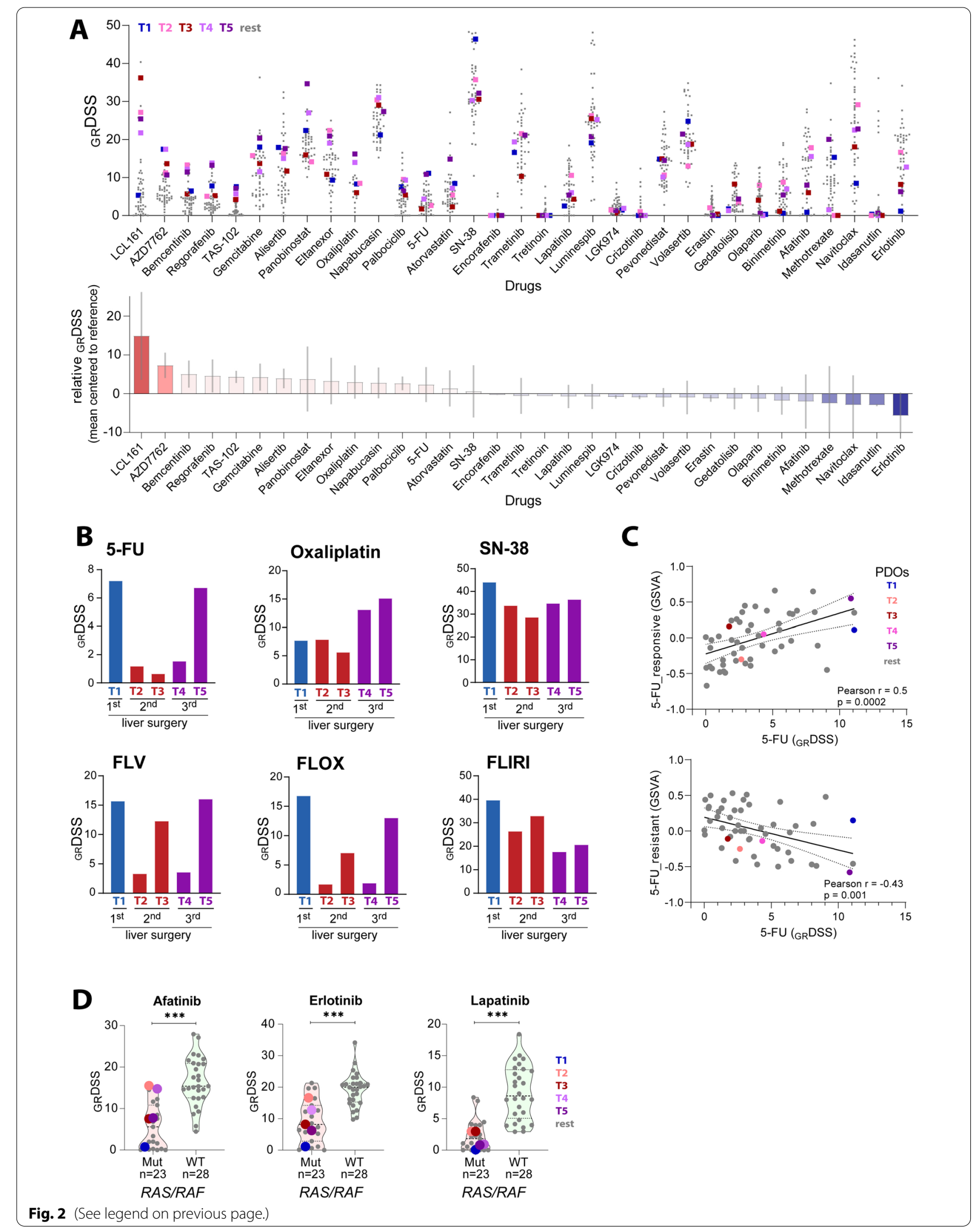


molecular hallmark of LCL161 activity is degradation of the targets c-IAP1, c-IAP2 and XIAP [19, 36, 37], and this was evident in post-treatment PDOs of all five lesions (Fig. 3B). Protein expression of XIAP and Survivin was highest in the resistant T1 PDOs, both after treatment with DMSO (control) and LCL161. In contrast, c-IAP1, c-IAP2 and XIAP were almost completely degraded after LCL161 treatment in the sensitive T2-T5 PDOs (Fig. 3B). Furthermore, treatment with LCL161 resulted in induction of apoptosis in a concentrationand time-dependent manner in the sensitive T3 PDOs, but not in the T1 PDOs, as indicated by increased PARP-, Caspase 7-, Caspase 8 and Caspase 3 activation (Fig. 3C).

Important mediators of TNF alpha signaling such as TNF, TNFRSF12A, three RIPK gene family members and genes encoding other apoptosis regulators such as CASP9, BAK1 and BIRC3 had expression levels positively correlated with LCL161 sensitivity across the T1-T5 and reference PDO dataset collectively (Fig. 3D). Strongest correlation with LCL161 activity was found for RIPK1 (Pearson $\mathrm{r}=0.6, \mathrm{p}<0.0001$ ), which also showed significant correlation among the T1-T5 PDOs separately (Pearson $r=0.92, p=0.03$ ). Furthermore, GSVA scores of the "Hallmark" gene set collection showed enrichment with the "TNFA signaling via NFKB" signature in all five PDOs from this patient, compared to the reference PDO dataset (Fig. 3E). This signature was also significantly associated with the gene sets of epithelial-mesenchymal transition, TGF $\beta$, upregulated KRAS, inflammation, and apoptosis, both in liver metastasis tissue samples [9] and corresponding PDOs, suggesting molecular interactions of the pathways (Fig. 3F). Notably, LCL161 sensitivity in PDOs was significantly correlated with GSVA scores of almost all of these gene sets (Fig. 3E), and most strongly with inflammatory response (Parsons's $\mathrm{r}=0.44, \mathrm{p}=0.0012$ ).

\section{Discussion}

Stratification of cancer patients according to molecular and functional tumor characteristics can improve treatment outcomes [17]. Here, we employed a multidisciplinary approach to analyze spatio-temporal pharmacogenomic heterogeneity in a patient with recurrent, KRAS mutated liver metastases from rectal cancer. Ex vivo co-clinical analyses of standard, neoadjuvant combination chemotherapies at three consecutive liver resections modeled the dynamics of clinical treatment responses, including indications of acquired resistance to FLOX. Furthermore, a switch in systemic treatment from FLOX to FLIRI corresponded to a higher ex vivo activity of oxaliplatin, indicating re-sensitization.

KRAS mutation in a microsatellite stable background and an undifferentiated histopathology are parameters associated with an unfavorable patient prognosis and limited systemic treatment options after development of resistance to standard therapies [18]. This case report indicated vulnerability to the experimental SMAC mimetic LCL161, showing higher drug activity in the recurrent liver metastases. LCL161 sensitivity patterns were supported by mechanistic analyses of the drug targets and TNF- $\alpha$ signaling, indicating target engagement and a potential for response prediction by RIPK1 gene expression levels, consistent with previously published clinical data in breast cancer [38]. Furthermore, IAP inhibitors can induce anti-tumor immunity [39, 40], and the strong inflammatory response in T1-T5 PDOs supports potency of a combination treatment of LCL161 and immune modulatory drugs. Combination with anti-PD1 therapy is currently being tested in a phase I study in patients with CRC (ClinicalTrials.gov Identifier: NCT02890069).

In conclusion, this case report supports that ex vivo pharmacotranscriptomics can model longitudinal treatment efficacy in metastatic CRC. It also supports further investigation of LCL161 as an anticancer agent,

\footnotetext{
(See figure on next page.)

Fig. 3 Anticancer activities of LCL161. A LCL161 GR DSS and IC 50 distribution in T1-T5 PDOs. Reference PDOs are indicated in grey. B Protein expression of IAPs analyzed with Western blotting after treatment with DMSO (-) and $200 \mathrm{nM} \mathrm{LCL161} \mathrm{(+)} \mathrm{for} 48 \mathrm{~h}$. C Time dependent effects of $200 \mathrm{nM}$ (lo) and $2000 \mathrm{nM}$ (hi) LCL161 on PARP cleavage, inhibition of XIAP and C-IAP1, Caspase- 8, 7 and 3 cleavage in LCL161-resistant T1 and sensitive T3 PDOs. (D, upper panel) Pearson correlation of significantly associating genes of RIP kinase family, gene members of the TNF signaling and other apoptosis regulators with ${ }_{\text {RR }} \mathrm{DSS}$ of LCL161. (D, lower panel) Scatter plot of RIPK1 gene expression versus LCL161 drug activity in PDOs. E. Enriched "Hallmark" gene sets in T1-T5 PDOs (colored) as compared to the reference PDO lines n=46 (grey). F Scatter plot of Pearson's correlation coefficients ( $r$ ) between the "TNFA signaling via NFKB" gene set and remaining 49"Hallmark" gene sets analyzed in 51 PDO lineages (vertical axis) and 30 liver metastasis tissue samples (horizontal axis). Highlighted in red are the gene sets that are enriched in T1-T5 PDOs and significantly associated with LCL161 drug activity. ${ }_{G R} D S S$ Growth adjusted drug sensitivity scores, $I C_{50}$ the half maximal inhibitory concentration, $C_{\text {max }}$ the maximum serum concentration of a drug, kDa kilodalton, GSVA gene set variation analysis
} 


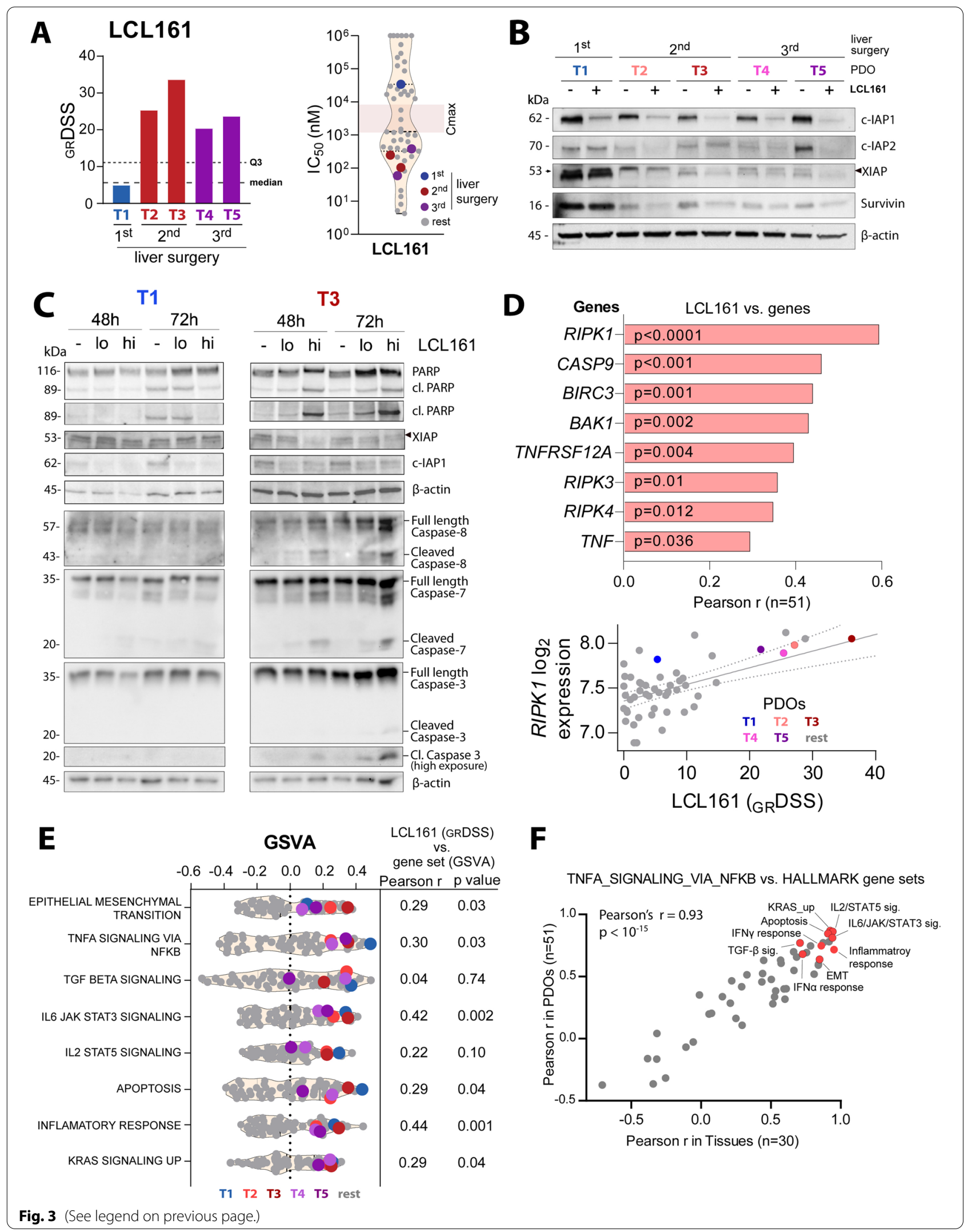




\section{although clinical translation of results from this case report was not possible due to the lack of ongoing stud- ies of IAP inhibitors in Norway.}

\begin{abstract}
Abbreviations
5-FU: 5-Fluorouracil; CDX2: Caudal type homeobox 2; CK20: Cytokeratin 20; CK7: Cytokeratin 7; CRC: Colorectal cancer; ECAD: E-cadherin; FLIRI: 5-Fluorouracil, leucovorin, and irinotecan; FLOX: 5-Fluorouracil, leucovorin, and oxaliplatin,; FLV: 5-Fluorouracil and leucovorin,; ${ }_{G R} D S S$ : Growth adjusted drug sensitivity scores; GSVA: Gene set variation analysis; IAP: Inhibitor of apoptosis protein; MRI: Magnetic resonance imaging; NF-kB: Nuclear factor kappa-B; PDO: Patient-derived organoid; RIPK1: Receptor-interacting protein kinase 1; SMAC: Second mitochondria-derived activator of caspases; TNF: Tumor necrosis factor
\end{abstract}

\section{Acknowledgements}

We are immensely grateful to the patient who donated the samples for the study and acknowledge Magdalena Kowalewska-Harbiyeli at the Department of Hepato-Pancreato-Biliary Surgery, OUH, for sample collection. We would like to thank Jarle Bruun, Christer Andreassen and Barbara Niederdorfer at the Department of Molecular Oncology, OUH, for their technical assistance.

\section{Authors' contributions}

RAL, KK and AS designed the study; KK, MZT conducted experiments; $K L$, AN AA, KK, SHM, MGG and MZT, assisted with material and data collection; KK, RAL, AS, SHM, PWE and CHB analyzed and interpreted data, KK, AS, and RAL wrote the manuscript. All authors read and approved the final manuscript.

\section{Funding}

This work was supported by grants from the Norwegian Cancer Society [project numbers 182759-2016 to RAL, 208336-2019 to AS, and 190188-2017 to MGG], the South-Eastern Norway Regional Health Authority [project numbers 2016123 to RAL, 2017102 to RAL and 2019042 to AS], and the Research Council of Norway [project numbers 250993 to RAL and 287899 to AS].

\section{Availability of data and materials}

All datasets analyzed during the current study are available from the corresponding author on reasonable request.

\section{Declarations}

\section{Ethics approval and consent to participate}

The study has been approved by the Norwegian Data Protection Authority and the Regional Committee for Medical and Health Research Ethics, SouthEastern Norway (reference 2010/1805 and 2017/780). Sample collection and research biobanks were conducted after obtaining written informed consent in accordance with the Declaration of Helsinki and national legislation.

\section{Consent for publication}

All authors approved the final manuscript for publication.

\section{Competing interests}

The authors declare no potential conflicts of interest.

\author{
Author details \\ ${ }^{1}$ Department of Molecular Oncology, Institute for Cancer Research, Oslo \\ University Hospital, Nydalen, P. O. Box 4953, 0424 Oslo, Norway. ${ }^{2}$ Institute \\ for Clinical Medicine, Faculty of Medicine, University of Oslo, Oslo, Norway. \\ ${ }^{3}$ Department of Oncology, Oslo University Hospital, Oslo, Norway. ${ }^{4}$ Depart- \\ ment of Hepato-Pancreato-Biliary Surgery, Oslo University Hospital, Oslo, \\ Norway. ${ }^{5}$ Institute of Clinical Medicine, University of Tromsø, Tromsø, Norway. \\ ${ }^{6}$ Division of Radiology and Nuclear Medicine, Oslo University Hospital, Oslo, \\ Norway. ${ }^{7}$ Department of Gastrointestinal Surgery, Oslo University Hospital, \\ Oslo, Norway.
}

Received: 28 June 2021 Accepted: 31 August 2021 Published online: 08 September 2021

\section{References}

1. van de Wetering $M$, Francies HE, Francis JM, Bounova G, lorio F, Pronk A, van Houdt W, van Gorp J, Taylor-Weiner A, Kester L, et al. Prospective derivation of a living organoid biobank of colorectal cancer patients. Cell. 2015;161:933-45.

2. Roerink SF, Sasaki N, Lee-Six H, Young MD, Alexandrov LB, Behjati S, Mitchell TJ, Grossmann S, Lightfoot H, Egan DA, et al. Intra-tumour diversification in colorectal cancer at the single-cell level. Nature. 2018;556:457-62.

3. Schumacher D, Andrieux G, Boehnke K, Keil M, Silvestri A, Silvestrov M, Keilholz U, Haybaeck J, Erdmann G, Sachse C, et al. Heterogeneous pathway activation and drug response modelled in colorectal-tumor-derived 3D cultures. PLoS Genet. 2019;15:e1008076.

4. Fujii M, Shimokawa M, Date S, Takano A, Matano M, Nanki K, Ohta Y, Toshimitsu K, Nakazato Y, Kawasaki K, et al. A colorectal tumor organoid library demonstrates progressive loss of niche factor requirements during tumorigenesis. Cell Stem Cell. 2016;18:827-38.

5. Weeber F, van de Wetering M, Hoogstraat M, Dijkstra KK, Krijgsman O, Kuilman T, Gadellaa-van Hooijdonk CG, van der Velden DL, Peeper DS, Cuppen $E P$, et al. Preserved genetic diversity in organoids cultured from biopsies of human colorectal cancer metastases. Proc Natl Acad Sci USA. 2015;112:13308-11.

6. Vlachogiannis G, Hedayat S, Vatsiou A, Jamin Y, Fernandez-Mateos J, Khan K, Lampis A, Eason K, Huntingford I, Burke R, et al. Patient-derived organoids model treatment response of metastatic gastrointestinal cancers. Science. 2018;359:920-6.

7. Ooft SN, Weeber F, Dijkstra KK, McLean CM, Kaing S, van Werkhoven E, Schipper L, Hoes L, Vis DJ, van de Haar J, et al. Patient-derived organoids can predict response to chemotherapy in metastatic colorectal cancer patients. Sci Transl Med. 2019;11:1.

8. Dagogo-Jack I, Shaw AT. Tumour heterogeneity and resistance to cancer therapies. Nat Rev Clin Oncol. 2018;15:81-94.

9. Bruun J, Kryeziu K, Eide PW, Moosavi SH, Eilertsen IA, Langerud J, Rosok B, Totland MZ, Brunsell TH, Pellinen T, et al. Patient-derived organoids from multiple colorectal cancer liver metastases reveal moderate intra-patient pharmacotranscriptomic heterogeneity. Clin Cancer Res. 2020;26:4107-19.

10. Pietrantonio F, Vernieri C, Siravegna G, Mennitto A, Berenato R, Perrone F, Gloghini A, Tamborini E, Lonardi S, Morano F, et al. Heterogeneity of Acquired Resistance to Anti-EGFR monoclonal antibodies in patients with metastatic colorectal cancer. Clin Cancer Res. 2017:23:2414-22.

11. Russo M, Siravegna G, Blaszkowsky LS, Corti G, Crisafulli G, Ahronian LG, Mussolin B, Kwak EL, Buscarino M, Lazzari L, et al. Tumor heterogeneity and lesion-specific response to targeted therapy in colorectal cancer. Cancer Discov. 2016;6:147-53.

12. Siravegna G, Mussolin B, Buscarino M, Corti G, Cassingena A, Crisafulli G, Ponzetti A, Cremolini C, Amatu A, Lauricella C, et al. Clonal evolution and resistance to EGFR blockade in the blood of colorectal cancer patients. Nat Med. 2015;21:795-801.

13. Nikbakht $H$, Jessa $S$, Sukhai MA, Arseneault M, Zhang T, Letourneau L, Thomas M, Bourgey M, Roehrl MHA, Eveleigh R, et al. Latency and interval therapy affect the evolution in metastatic colorectal cancer. Sci Rep. 2020;10:581.

14. Siravegna $G$, Bardelli A. Failure is not final: ctDNA-guided rechallenge therapy in colorectal cancer. Ann Oncol. 2019;30:1671.

15. Parseghian CM, Loree JM, Morris VK, Liu X, Clifton KK, Napolitano S, Henry JT, Pereira AA, Vilar E, Johnson B, et al. Anti-EGFR-resistant clones decay exponentially after progression: implications for anti-EGFR re-challenge. Ann Oncol. 2019:30:243-9.

16. Pauli C, Hopkins BD, Prandi D, Shaw R, Fedrizzi T, Sboner A, Sailer V, Augello M, Puca L, Rosati R, et al. Personalized In Vitro and In Vivo Cancer Models to Guide Precision Medicine. Cancer Discov. 2017;7:462-77.

17. Sveen A, Kopetz S, Lothe RA. Biomarker-guided therapy for colorectal cancer: strength in complexity. Nat Rev Clin Oncol. 2020;17:11-32.

18. Van Cutsem E, Cervantes A, Adam R, Sobrero A, Van Krieken JH, Aderka D, Aranda Aguilar E, Bardelli A, Benson A, Bodoky G, et al. ESMO consensus guidelines for the management of patients with metastatic colorectal cancer. Ann Oncol. 2016;27:1386-422.

19. Gyrd-Hansen M, Meier P. IAPs: from caspase inhibitors to modulators of NF-kappaB, inflammation and cancer. Nat Rev Cancer. 2010;10:561-74. 
20. Flanagan L, Kehoe J, Fay J, Bacon O, Lindner AU, Kay EW, Deasy J, McNamara DA, Prehn JH. High levels of $X$-linked Inhibitor-of-Apoptosis Protein (XIAP) are indicative of radio chemotherapy resistance in rectal cancer. Radiat Oncol. 2015;10:131

21. Darding M, Meier P. IAPs: guardians of RIPK1. Cell Death Differ. 2012;19:58-66.

22. Deveraux QL, Takahashi R, Salvesen GS, Reed JC. X-linked IAP is a direct inhibitor of cell-death proteases. Nature. 1997;388:300-4.

23. Mifflin L, Ofengeim D, Yuan J. Receptor-interacting protein kinase 1 (RIPK1) as a therapeutic target. Nat Rev Drug Discov. 2020;19:553-71.

24. Morrish E, Brumatti G, Silke J. Future therapeutic directions for smacmimetics. Cells. 2020;9:1.

25. Sveen A, Bruun J, Eide PW, Eilertsen IA, Ramirez L, Murumagi A, Arjama M, Danielsen SA, Kryeziu K, Elez E, et al. Colorectal cancer consensus molecular subtypes translated to preclinical models uncover potentially targetable cancer cell dependencies. Clin Cancer Res. 2018;24:794-806.

26. Yadav B, Pemovska T, Szwajda A, Kulesskiy E, Kontro M, Karjalainen R, Majumder MM, Malani D, Murumagi A, Knowles J, et al. Quantitative scoring of differential drug sensitivity for individually optimized anticancer therapies. Sci Rep. 2014;4:5193.

27. Hafner M, Niepel M, Chung M, Sorger PK. Growth rate inhibition metrics correct for confounders in measuring sensitivity to cancer drugs. Nat Methods. 2016;13:521-7.

28. Brunsell TH, Sveen A, Bjornbeth BA, Rosok BI, Danielsen SA, Brudvik KW, Berg KCG, Johannessen B, Cengija V, Abildgaard A, et al. High concordance and negative prognostic impact of RAS/BRAF/PIK3CA mutations in multiple resected colorectal liver metastases. Clin Colorectal Cancer. 2020;19:e26-47.

29. Gautier L, Cope L, Bolstad BM, Irizarry RA. affy-analysis of Affymetrix GeneChip data at the probe level. Bioinformatics. 2004;20:307-15.

30. Sandberg R, Larsson O. Improved precision and accuracy for microarrays using updated probe set definitions. BMC Bioinform. 2007;8:48.

31. Schutte M, Risch T, Abdavi-Azar N, Boehnke K, Schumacher D, Keil M, Yildiriman R, Jandrasits C, Borodina T, Amstislavskiy V, et al. Molecular dissection of colorectal cancer in pre-clinical models identifies biomarkers predicting sensitivity to EGFR inhibitors. Nat Commun. 2017;8:14262.

32. Subramanian A, Tamayo P, Mootha VK, Mukherjee S, Ebert BL, Gillette MA et al (2005) Gene set enrichment analysis: a knowledgebased approach for interpreting genome-wide expression profiles. Proc Natl Acad Sci U S A 102(43):15545-15550. https://doi.org/10.1073/pnas.0506580102

33. Hanzelmann S, Castelo R, Guinney J. GSVA: gene set variation analysis for microarray and RNA-seq data. BMC Bioinform. 2013;14:7.

34. McCann C, Matveeva A, McAllister K, Van Schaeybroeck S, Sessler T, Fichtner M, Carberry S, Rehm M, Prehn JHM, Longley DB: Development of a protein signature to enable clinical positioning of IAP inhibitors in colorectal cancer. FEBS J 2021.

35. Eisenhauer EA, Therasse P, Bogaerts J, Schwartz LH, Sargent D, Ford R, Dancey J, Arbuck S, Gwyther S, Mooney M, et al. New response evaluation criteria in solid tumours: revised RECIST guideline (version 1.1). Eur J Cancer. 2009;45:228-47.

36. Infante JR, Dees EC, Olszanski AJ, Dhuria SV, Sen S, Cameron S, Cohen RB. Phase I dose-escalation study of LCL161, an oral inhibitor of apoptosis proteins inhibitor, in patients with advanced solid tumors. J Clin Oncol. 2014;32:3103-10.

37. Vince JE, Wong WW, Khan N, Feltham R, Chau D, Ahmed AU, Benetatos CA, Chunduru SK, Condon SM, McKinlay M, et al. IAP antagonists target CIAP1 to induce TNFalpha-dependent apoptosis. Cell. 2007;131:682-93.

38. Bardia A, Parton M, Kummel S, Estevez LG, Huang CS, Cortes J, Ruiz-Borrego M, Telli ML, Martin-Martorell P, Lopez R, et al: Paclitaxel With Inhibitor of Apoptosis Antagonist, LCL161, for Localized Triple-Negative Breast Cancer, Prospectively Stratified by Gene Signature in a Biomarker-Driven Neoadjuvant Trial. J Clin Oncol 2018; 1: JCO2017748392.

39. Beug ST, Beauregard CE, Healy C, Sanda T, St-Jean M, Chabot J, Walker DE, Mohan A, Earl N, Lun X, et al. Smac mimetics synergize with immune checkpoint inhibitors to promote tumour immunity against glioblastoma. Nat Commun. 2017;8:1.

40. Chesi M, Mirza NN, Garbitt VM, Sharik ME, Dueck AC, Asmann YW, Akhmetzyanova I, Kosiorek HE, Calcinotto A, Riggs DL, et al. IAP antagonists induce anti-tumor immunity in multiple myeloma. Nat Med. 2016;22:1411-20.

\section{Publisher's Note}

Springer Nature remains neutral with regard to jurisdictional claims in published maps and institutional affiliations.
Ready to submit your research? Choose BMC and benefit from:

- fast, convenient online submission

- thorough peer review by experienced researchers in your field

- rapid publication on acceptance

- support for research data, including large and complex data types

- gold Open Access which fosters wider collaboration and increased citations

- maximum visibility for your research: over 100M website views per year

At BMC, research is always in progress.

Learn more biomedcentral.com/submissions 